\title{
Species discrimination in plasma welding spectra by means of Principal and Independent Component Analysis
}

\author{
E. Real*, J. Mirapeix, O. M. Conde, R. Ruiz-Lombera, A. Cobo, J.M. Lopez-Higuera \\ Photonics Engineering Group (University of Cantabria), Plaza de la Ciencia s/n, 39005, \\ Santander (Cantabria), Spain
}

\begin{abstract}
Principal and Independent Component Analysis are used in this paper to provide a discrimination among those species participating in the plasma of welding spectra. This approach might be useful for spectral line identification for emission spectroscopy, especially for online welding diagnostics and laser induced breakdown spectroscopy. In this case, the feasibility of this proposal will be analyzed by means of arcwelding experiments where different plasma species will be separated by the proposed processing scheme.
\end{abstract}

Keywords: Independent Component Analysis, Principal Component Analysis, plasma spectroscopy, welding diagnostics, laser induced breakdown spectroscopy, optical fiber, CCD spectrometer

\section{INTRODUCTION}

Identification of emission lines in spectra derived from different applications is still a problem without a definitive solution. The typical procedure followed to associate a species (element and ionization stage) with a given emission line is to search within specific databases with spectroscopic information, using the central wavelength of the line under analysis as the main identification parameter, but also another spectroscopic parameters such as the emission line relative intensity. Some authors have proposed more sophisticated approaches, based on information retrieval techniques ${ }^{1}$ or linear or rank correlation ${ }^{2}$, but it seems that fields of application such as laser induced breakdown spectroscopy (LIBS) or plasma optical spectroscopy applied to welding diagnostics, still need an improved solution to solve the emission line identification problem.

Within this scenario, some algorithms able to analyze the information regarding correlation or independency among the data under analysis have been proposed by different authors. For example, Yu et al. ${ }^{3}$ use PCA on plasma spectra captured from arc welding, obtaining two principal components with mixed information of gas and metallic lines. PCA has also been used for laser welding monitoring based on the analysis of near-infrared images ${ }^{4}$; and a combination of PCA and ICA has been employed ${ }^{5}$ to perform a blind source separation of different acoustic signals in an industrial environment within the framework of welding monitoring by means of acoustic monitoring.

In this paper a solution based on the employment of PCA and ICA is proposed to help in the identification of the different emission lines of the plasma spectra: in particular to carry out a species discrimination.

\section{SPECTRAL PROCESSING SCHEME}

PCA and ICA procedures are two different cases of blind signal separation, what might prove suitable for the identification of the plasma spectral species discussed above. These algorithms are intended to separate a set of relevant signals from a set of mixed signals without any other information than the mixed data.

*eusebio.real@unican.es; phone (+34) 942 200877; fax (+34) 942200877

23rd International Conference on Optical Fibre Sensors, edited by José Miguel López-Higuera,

Julian Jones, Manuel López-Amo, José Luis Santos, Proc. of SPIE Vol. 9157, 91570F

(C) 2014 SPIE · CCC code: $0277-786 X / 14 / \$ 18 \cdot$ doi: $10.1117 / 12.2054722$ 
PCA is a statistical technique used to transform a set of variables into a new set of linearly uncorrelated variables, called principal components (PC). The resultant coordinate system has its coordinates ordered from high to low variance in the original data. This procedure separates the original data set into a new data set where variables are not only uncorrelated but also ordered according to their variance in the original data.

ICA is a different statistical technique designed to obtain independent variables instead of just uncorrelated as it happens with PCA. This procedure is based on the assumption that the data are formed by an additive composition of independent signals, called independent components (IC). In this paper ICA is implemented by means of the FastICA algorithm ${ }^{6}$.

The advantage of ICA over PCA lies in the extraction of independent components rather than uncorrelated. However, the ICA outputs are not ordered according to any criteria, so that the most relevant components could be in any position. In the present study, PCA is used to decrease the number of spectra prior to applying ICA. Only the most relevant components of PCA, according to a dynamic selection criterion $^{7}$, are considered for ICA processing. The independent components resulting from ICA are corrected in sign and also have their mean subtracted in order to identify peaks in the spectra under analysis. The final identification of species is performed by comparing the most relevant peaks of each independent component with the peaks of known species, such as Fe I or Ar II.

\section{RESULTS AND DISCUSSION}

To verify the suitability of the proposed solution several arc-welding (Gas Tungsten Arc Welding: GTAW) experiments were performed with the welding setup described in a previous work ${ }^{8}$. In terms of the optical setup, two optical fibers were employed to guide the plasma radiation from the vicinity of the weld pool to the chosen CCD spectrometers, with nominal resolutions of 0.03 and $0.3 \mathrm{~nm}$, and spectral ranges of approximately 180 to $530 \mathrm{~nm}$ and 370 to $500 \mathrm{~nm}$, respectively. AISI 304 stainless-steel plates were used for the tests, where both correct welds and seams with different defects were generated.

Given that the appearance of defects gives rise to variations in the plasma spectra and this might be useful for the proposed solution, a seam with two defects has been selected to be discussed in this paper (Fig. 1).

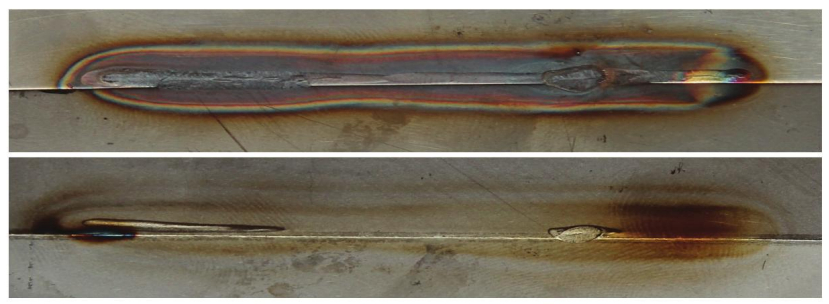

Figure 1. Top and bottom sides of the seam under analysis.

The spectral data captured during the process were used as input for a processing scheme with two stages based on applying PCA and, afterwards, ICA. The results in terms of the first independent components (IC) are shown in Figure 2, where a sample of the acquired plasma spectra has also been depicted. The first IC, IC\#1, exhibits to main lines at $\lambda \approx 393.4$ and $396.7 \mathrm{~nm}$, associated with Fe I. In this case, this association is somewhat easy, given that these Fe I lines are predominant in that spectral range, and there are two lines with significant relative intensities ${ }^{9}$ close to those wavelengths.

IC\#2 shows more lines, being the three most intense located at $\lambda \approx 425.3,427.3$ and $428.8 \mathrm{~nm}$; but also with lines at $\lambda \approx 393.4,396.7,404.4$ and $438.3 \mathrm{~nm}$. Given the chemical composition of AISI 304 (with Fe between 66 and 74\%, Cr between 18 and 20\%, Ni between 8 and $10.5 \%$ and $\mathrm{Mn}$ up to 2\%), the lines with wavelengths between 425 and 429 might be associated with $\mathrm{Cr}$ I. However, given that the other lines considered in IC\#2 can be associated with Fe I, and that there are also Fe I lines with high relative 
intensities in those other wavelengths, it might be concluded that IC\#2 can be also associated with Fe I. The lines included in IC\#3 can be all of them associated with Ar II, with wavelengths at $\lambda \approx 434.8,440.1$, $459,461,472.7,473.6,480.6,484.8$ and $488 \mathrm{~nm}$. It can be observed that the different ICs seem to be able to discriminate among the different species participating in the plasma, what can be of great interest for line identification purposes.

Regarding the performance of a single stage implementing PCA (without the assistance of ICA) Figure 3(b) shows an example of the first PC component obtained for the same experiment under analysis. It can be appreciated how it appears to be less discriminative in terms of species separation than the previous solution. To better clarify this point, Figure 3(a) depicts the correlation map between the main PCs and ICs. Given that the correlation is generally low, it can be deduced that ICA is able to extract meaningful information from the majority of PCs, thus given rise to ICs with less spectral lines. There are some cases, for example for PC\#10 and IC\#2, where the correlation is high (approximately 0.8), what implies that, even in this case, the spectral information contained in both components will differ.
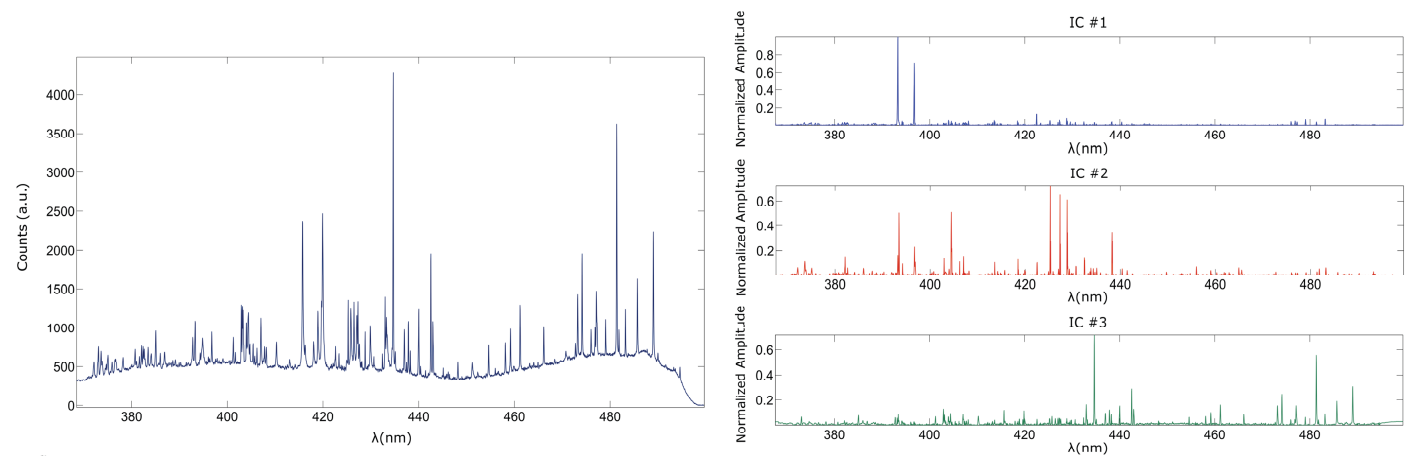

Figure 2. Plasma spectrum captured during the welding of the seam shown in Fig. 1 (left). Three main independent components (ICs) associated with Fe I and Ar II species (right).

a)
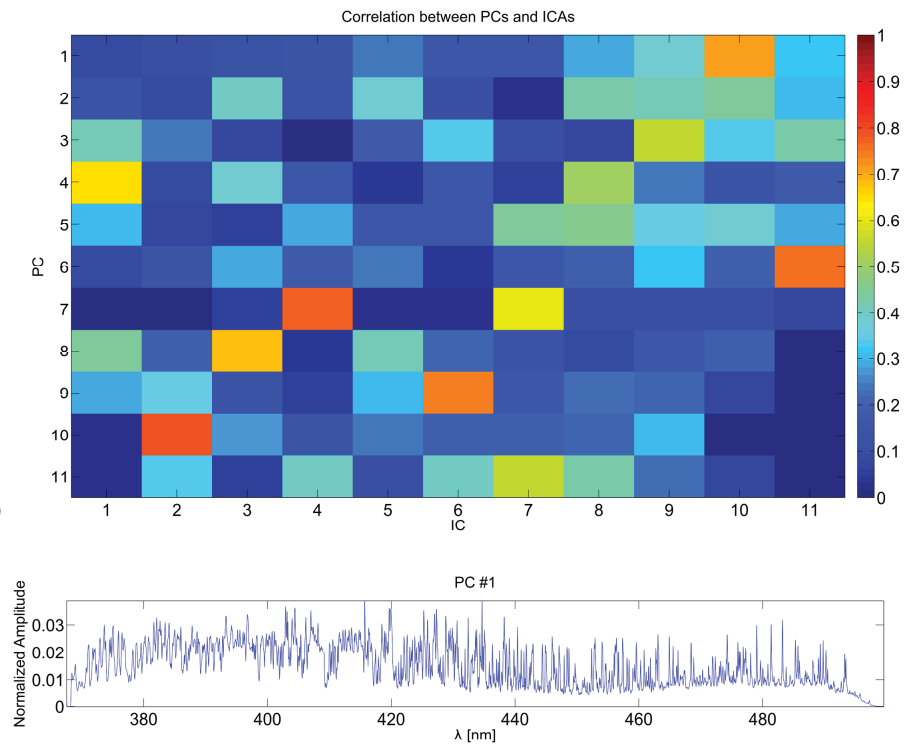

b)

Figure 3. (a) Correlation map of Principal Components (PCs) and Independent Components (ICs). (b) First PC obtained for the seam shown in Fig. 1. 


\section{CONCLUSIONS}

A solution based on the use of Principal Component Analysis (PCA) and Independent Component Analysis (ICA) for species discrimination of emission spectra has been discussed in this paper. By employing ICA after PCA, the resulting components exhibit lines belonging to the same species, thus proving that ICA is able to discriminate among species by detecting the different behavior of the emission lines belonging to the same species during the process. Although this proposal would be also of interest in other research areas, such as LIBS, in this case arc-welding monitoring has been chosen as the experimental scenario. Analyses with different materials will be performed, as well as an exploration of the usefulness of the scores provided by these algorithms to be used as indicators of the participation of each species within the plasma.

\section{ACKNOWLEDGEMENTS}

This work has been supported by the project TEC2010-20224-C02-02 and FIS2010-19860.

\section{REFERENCES}

[1] Amato, G., Cristoforetti, G., Legnaioli, S., Lorenzetti, G., Palleschi, V., Sorrentino, F., and Tognoni, E., "Progress towards an unassisted element identification from Laser Induced Breakdown Spectra with automatic ranking techniques inspired by text retrieval," Spectrochimica Acta Part B: Atomic Spectroscopy, 65(8), 664-670 (2010).

[2] Gornushkin, I., Mueller, M., Panne, U., and Winefordner, J., "Insights into linear and rank correlation for material identification in laser-induced breakdown spectroscopy and other spectral techniques," Applied Spectroscopy, 62(5), 542-553 (2008).

[3] Yu, H., Ye, Z., and Chen, S., "Application of arc plasma spectral information in the monitor of $\mathrm{Al}-\mathrm{Mg}$ alloy pulsed GTAW penetration status based on fuzzy logic system," The International Journal of Advanced Manufacturing Technology, 68, 2713-2727 (2013).

[4] Xiangdong, G., and Qian, W., "Monitoring of high-power fiber laser welding based on principal component analysis of a molten pool configuration," Laser Physics, 23(12), 126001 (2013).

[5] Sansan, A., Zhen, L., Nan, Z., and Rui, W., "Blind Source Separation based on Principal Component Analysis-Independent Component Analysis for Acoustic Signal during Laser Welding Process," International Conference on Digital Manufacturing and Automation (ICDMA) 1, 336-339 (2010).

[6] Hyvärinen, A., and Oja, E., "Independent component analysis: algorithms and applications," Neural Networks, 13(4), 411-430 (2000).

[7] Eguizabal, A., Laughney, A. M., García-Allende, P. B., Krishnaswamy, V., Wells, W. A., Paulsen, K. D., Pogue, B. W., Lopez-Higuera, J. M., and Conde, O. M., "Direct identification of breast cancer pathologies using blind separation of label-free localized reflectance measurements," Biomedical Optics Express, 4(7), 1104-1118 (2013).

[8] Rodriguez-Cobo, L., Mirapeix, J., Ruiz-Lombera, R., Cobo, A., and Lopez-Higuera, J., "Fiber Bragg grating sensors for on-line welding diagnostics," 214(4), 839-843 (2014).

[9] Ralchenko, Y., Kramida, A., Reader, J., and Team, N. A. S. D., "NIST Atomic Spectra Database (version 3.1. 5)," National Institute of Standards and Technology, Gaithersburg, MD, (2008). 